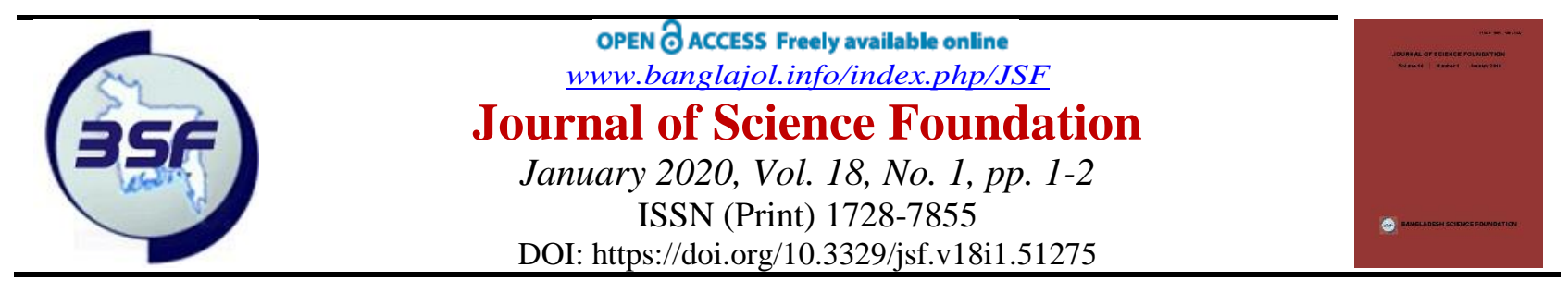

\title{
Editorial
}

\section{Impact of Climate Change in Rural Health: Bangladesh Perspective}

\author{
Zebunnesa Zeba $^{1}$
}

Climate change is increasing the intensity and variability of weather conditions, and this will have direct health impacts for people in rural and remote Bangladesh. Human activities particularly the burning of fossil fuels, have released enough carbon dioxide and other green-house gases to trap additional heat in the lower atmosphere and affect the global climate. Surface air temperature has warmed by $0.9^{\circ} \mathrm{C}$ since 1910 . Daytime maximum temperatures have warmed by $0.8^{\circ} \mathrm{C}$ over the same period, while overnight minimum temperatures have warmed by $1.1^{\circ} \mathrm{C}$. By 2070 , temperatures are projected to rise another 1.0 to $2.5^{\circ} \mathrm{C}$ if greenhouse gas emissions are seriously reduced, or 2.2 to $5.0^{\circ} \mathrm{C}$ if emissions are not reduced with inland temperature rises greater than on the coast (Houghton et al., 2017).

As a consequence, sea levels are rising, glaciers and icesheets are melting and precipitation patterns are changing. Extreme weather events such as heatwaves, floods, droughts and cyclones will become more frequent and intense. The vulnerability of communities to the health impacts of climate change is a function of a range of factors, such as age profile, pre-existing health conditions, awareness of the risks, socio-economic status, housing and the surrounding infrastructure such as communications, transport, water and sanitation, exposure of particular livelihoods, and access to health services. An unfavorable combination of these factors leaves many communities in rural and remote Bangladesh more vulnerable.

Heat-related illness and death is expected to increase, particularly in rural areas where the ageing of the population is more marked, there is a greater prevalence of chronic conditions, people live in more isolated physical and social circumstances, and homes are hotter. Older people are more vulnerable because of the physiological effects of ageing like declining cognitive state, reduced sweating response, greater prevalence of chronic conditions and disability, reduced mobility and the deleterious impact of prescribed medication on the body's ability to cope with higher temperatures.

People in rural and remote areas are more likely to be socially isolated because of sparse populations and the lack of public transport. They may also be less mobile personally due to their higher prevalence of chronic conditions. Poorer physical and personal mobility makes people more vulnerable to injury and death from drought, cyclone and flooding. Poor seasons which is a consequence of unfavourable weather conditions and extreme weather events and the subsequent stresses of lost income, mounting debt and damage to property which befall farmers and their families are likely to prompt a rise in mental illness and harmful behavioural responses. This is expected to surface in the form of depression and anxiety, psychological trauma and posttraumatic stress, drug and alcohol abuse, social withdrawal, relationship disharmony and, in extreme cases, self-harm and suicide (Walker 2019).

Drought, flooding and other extreme weather events can affect the price and availability of food, placing people at increased risk of malnutrition. Poor nutrition is associated with many disorders, including in relation to child growth and development and in later life. The availability of fresh food and vegetables declines with remoteness, and people living there are less likely to adhere to nutritional and dietary guidelines.

\footnotetext{
${ }^{1}$ Assistant Professor, Department of Public Health and Informatics, Jahangirnagar University, Savar, Dhaka-1342, Bangladesh; Email: zebunnesa.zeba@juniv.edu; Cell no.: +8801711106654
} 
Access to clean water is essential for drinking, personal hygiene, bathing and food preparation. However some communities in remote Bangladesh have no option other than to use water that is untreated or only partially treated - whether from a local water course, a bore or rooftop collection. Prolonged periods of drought will lead to greater reliance on such sources, which are more susceptible to contamination, particularly when raw water is of poor quality or when catchments are affected by events like flooding. Heavy rain and flooding can also cause contamination to such water supplies through mixing with agricultural pesticides and fertilizers, septic waste and animal carcases.

Warmer temperatures will increase bacterial growth which can lead to an increase in food-borne infections like salmonella. The impact will depend on factors such as food hygiene practices, and the homes in some remote communities lack the equipment and resources necessary to safely store and prepare foods like refrigerators, potable water supply and waste management. The transmission of certain arboviruses such as Dengue and Chikungunya viruses will also increase, prompted by environmental conditions that enable breeding and survival; rainfall, tides, sea level, temperature, humidity and wind all play a part. Therefore, there is a great impact of climate changes in rural community people of Bangladesh.

\section{References}

Houghton A, Austin J, Beerman A, Horton C. An approach to developing local climate change environmental public health indicators in a rural district. Journal of environmental and public health. 2017;2017

Walker J. Rural health inequities and the impact of climate change. Australian Journal of Rural Health. 2019;27(6):583-4

[Journal of Science Foundation, January 2020;18(1):1-2] 